\title{
ENTANGLEMENT IN SHOR'S FACTORING ALGORITHM
}

\author{
Jianing Tan ${ }^{1}$ Zhihao Liu ${ }^{1,2}$ and Hanwu Chen ${ }^{1,2}$ \\ ${ }^{1}$ School of Computer Science and Engineering, Southeast University, \\ Nanjing 210096, China \\ ${ }^{2}$ Key Laboratory of Computer Network and Information Integration in Southeast \\ University, Ministry of Education, Nanjing 210096, China
}

\begin{abstract}
Quantum algorithms are well known for their higher efficiency compared to their classical counterparts. However, the origin of the speed-up offered by quantum algorithms is a debatable question. Using entanglement measure based on coefficient matrix, we investigate the entanglement features of the quantum states used in Shor's factoring algorithm. The results show that if and only if the order $r$ is 1 , the algorithm generates no entanglement. Finally, compare with published studies results (Proceedings: Mathematical, Physical and Engineering Sciences, 459(2036): 2011-2032, 2003, Physical Review A, 72(6): 062308, 2005), we give counter examples to show that previous researches neglect partially entanglement.
\end{abstract}

\section{KEYWORDS}

Shor's factoring algorithm, entanglement measure, coefficient matrices

\section{INTRODUCTION}

Quantum algorithm is theoretically able to solve certain problem much more quickly than classical algorithm which is even the best currently known. The most well-known algorithms are Shor's algorithm and Grover's algorithm. The former runs exponentially faster than the best known classical algorithm for factoring. And the latter runs quadratically faster than the best possible classical algorithm for searching an unstructured database or an unordered list. While understanding of quantum algorithms is still incomplete, most researchers believe that entanglement is necessary to realize quantum computing. Algorithms such as Grover's algorithm [1-4], and Shor's factoring algorithm [5-7] are investigated. The results show that quantum entanglement as a fascinating nonclassical feature plays a crucial role in making algorithms powerful [8], especially for quantum algorithm operating on pure states [9]. However, there has also been much debate on the role of entanglement in making quantum algorithms powerful. Ref. [10] indicate that only quantum parallelism is the only tool required from quantum mechanics to overcome their classical counterparts, the role of entanglement has not been clear yet.

In this paper, we investigate multipartite entanglement in Shor's factoring algorithm. The entanglement measure we used is the measure based based on coefficient matrix [11]. Differ from Ref. [5], quantum states we focus are all the states (both including in the first register and the second register) the algorithm involved, but not states in the first register after the measurement. The results show that the algorithm will theoretically not generate entanglement in the case when 
the input state of the second register is an explicit eigenstate and the case when the order $r$ is equal to 1 . But in practical works, it will always generate entanglement.

The remainder of this paper is organized as follows. Sec.2 briefly introduces Shor's factoring algorithm and the entanglement measure we used. Then we analyze multipartite entanglement occurs in the algorithm in Sec. 3. Finally, some conclusions are draw in Sec.4.

\section{Preliminaries}

\subsection{Shor's factoring algorithm}

Given a positive composite integer $\mathrm{N}$, factoring problem is to find prime numbers when multiplied together equal $\mathrm{N}$. It turns out to be equivalent to the order-finding problem [12], which can be solved by quantum phase estimation algorithm. For positive integers $\mathrm{x}$ and $\mathrm{N}, \mathrm{x}<\mathrm{N}$, with no common factors, the order of $\mathrm{x}$ modulo $\mathrm{N}$ is defined to be the least positive integer, $\mathrm{r}$, such that $\mathrm{xr}=1(\bmod \mathrm{N})$. The order-finding problem is to determine the order for some specified $\mathrm{x}$ and $\mathrm{N}$ [12]. Fig. 1 shows quantum circuit representation of quantum phase estimation algorithm.

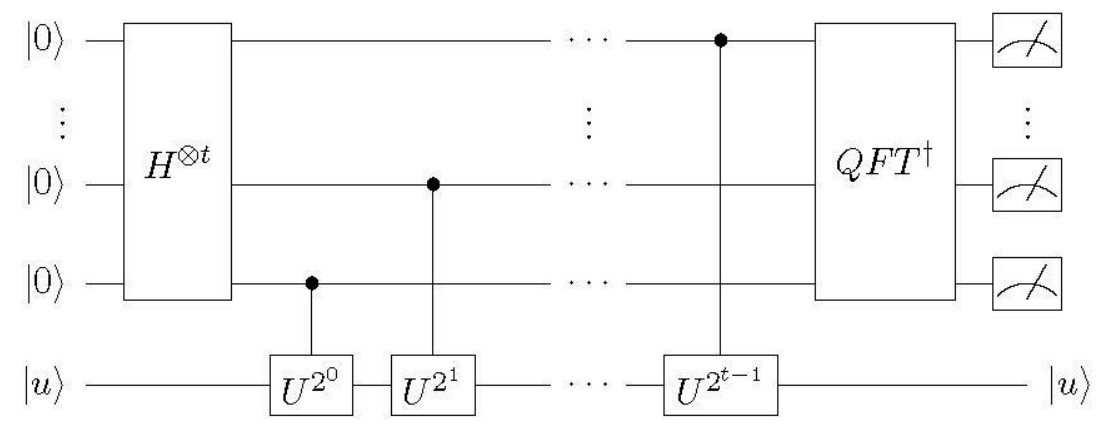

Figure 1: Quantum circuit representation of

quantum phase estimation algorithm. H: Hadamard gate. U: unitary operator. |u): an eigenvector of a unitary operator $\mathrm{U}$ with eigenvalue e2niV. $\mathrm{t}$ : the number of qubit in the first register. $\left(\mathrm{QFT}^{+}\right.$: inverse quantum fourier transform.

Usually, register for phase estimation is called first register, register for eigen state $\mathrm{u}$ ) is called second register. The algorithm is performed in two stages. The circuit before the $Q F T^{\dagger}$ is called the first stage, then the second stage is to apply the $Q F T^{\dagger}$ on the first register. Refs. [5, 7] indicate that entanglement remains nearly constant during the quantum fourier transform stage. And it is consistent with our experimental results. So in this paper, we focus on the first stage.

The quantum algorithm for order-finding is just the phase estimation algorithm applied to the unitary operator [12]

$$
U|y\rangle \equiv|x y(\bmod N)\rangle
$$

The states defined by

$$
\left|u_{s}\right\rangle \equiv \frac{1}{\sqrt{r}} \sum_{k=0}^{r-1} e^{\frac{-2 \pi i s k}{r}}\left|x^{k} \bmod N\right\rangle
$$


for integer $0 \leq s \leq r-1$ are eigenstates of $\mathrm{U}$, since

$$
U\left|u_{s}\right\rangle=e^{\frac{2 \pi i s}{r}}\left|u_{s}\right\rangle
$$

Using quantum phase estimation estimation algorithm allows us to obtain, with high accuracy, $\varphi=\frac{s}{r}$ . Then we can obtain the order $r$ easily. During the calculation process the final state resulting from the first stage of the algorithm is[12]

$\left|\psi_{\text {stage } 1}\right\rangle=\frac{1}{2^{t / 2}}\left(|0\rangle+e^{2 \pi i 2^{t-1} \varphi_{s}}|1\rangle\right)\left(|0\rangle+e^{2 \pi i 2^{t-2} \varphi_{s}}|1\rangle\right) \ldots\left(|0\rangle+e^{2 \pi i 2^{0} \varphi_{s}}|1\rangle\right)\left(\left|u_{s}\right\rangle\right)$

Obviously, the final state can be written as the form of product state. Shor's factoring algorithm does not generate entanglement.

In practical works, using phase estimation procedure rely on a restrictive condition that we must prepare $\left.\mid u_{s}{ }^{\prime}\right)$ for the second register. It is difficult to satisfy. Because it requires that we know r, so this is out of the question. Fortunately, we can prepare a superposition state of $\left(\mathrm{u}_{\mathrm{s}}{ }^{\prime}\right)$ without knowing $r$, which is that

$$
|1\rangle=\frac{1}{\sqrt{r}} \sum_{s=0}^{r-1}\left|u_{s}\right\rangle
$$

In Sec. 3, we will prove, Shor's factoring algorithm will generate entanglement when the input quantum state of the second register is a superposition state of the eigen states.

\subsection{Entanglement Measure Based on Coefficient Matrices}

Entanglement is an attribute unique to quantum systems. The concept of it enters some deep investigations of quantum information and computation. Ref. [13] demonstrates that entanglement is a necessary condition for an exponential computational speedup with respect to classical computations. Peng Xue et al. proved that entanglement is a particular manifestation of a more fundamental quantum resource-both contextuality and nonlocality can consume this resource [14]. Entanglement quantification is referred to as entanglement measure. Thus, multiqubit states often have difficulty obtaining the entanglement value. Numerous entanglement measures have been defined for the different properties of a state. In recent decades, coefficient matrix draws the attention of researchers. Because the rank of it is an inherent physical property since it is invariant under SLOCC [15]. It is used to classify multiqubit states $[15,16]$ and then a entanglement measure [11] is proposed based on it. Compare with other entanglement measures, this measure is easy to calculate and capable of dealing with quantum pure states with arbitrary dimensions and parties. 
Let $d_{t}$ is the dimension of each subsystem, $\left|i_{1}, \ldots, i_{N}\right\rangle$ are the standard basis states, and $c_{i_{1}, \ldots, i_{N}}$

are the coefficients. We can write an $\mathrm{N}$-qudit pure state as

$$
|\psi\rangle_{1 \ldots N}=\sum_{i_{1}, \ldots, i_{N}=0}^{\left(d_{1}-1\right), \ldots,\left(d_{N}-1\right)} c_{i_{1}, \ldots, i_{N}}\left|i_{1}, \ldots, i_{N}\right\rangle
$$

The coefficient matrix is defined as follow [11]

$$
\left(\begin{array}{ccc}
c_{0 \cdots 0,0 \cdots 0} & \cdots & c_{0 \cdots 0, \underbrace{d_{l}-1 \cdots d_{N}-1}_{l}} \\
l \quad \underbrace{}_{N-l} & & c_{0 \cdots 1, \underbrace{d_{l}-1 \cdots d_{N}-1}_{N-l}} \\
c_{0 \cdots 1,0 \cdots 0} & \cdots & \vdots \\
l \quad N-l & \vdots & c_{l}^{c_{d_{1}-1 \cdots d_{l}-1}}, \underbrace{d_{l}-1 \cdots d_{N}-1}_{N-l}
\end{array}\right)
$$

where bits 1 to 1 and $1+1$ to $\mathrm{N}$ are referred to as the row bits and column bits respectively. Based on coefficient matrix, Ref. [11] propose a entanglement measure and proof it is a good entanglement measure. The mathematical expression of the bipartite entanglement measure is

$$
E_{C o e}(|\psi\rangle)=\sqrt{\sum_{i<j} 4\left(\left\|x_{i}\right\|^{2}\left\|x_{j}\right\|^{2}-\left|\left(x_{i} \cdot x_{j}\right)\right|^{2}\right)}
$$

here $\mathrm{x}_{\mathrm{i}}\left(\mathrm{x}_{\mathrm{j}}\right)$ represents the $\mathrm{ith}(\mathrm{jth})$ row vector of coefficient matrix, $\left\|\mathrm{x}_{\mathrm{i}}\right\|$ denotes the length of the vector $\mathrm{xi}$, and $|\mathrm{xi}|$ is the module of $\mathrm{xi}$. Then general way of characterizing the global amount of entanglement exhibited by an $n$-qubit state is provided by the sum of the bipartite entanglement measures associated with the $2^{n-1}-1$ possible bipartitions of the n-qubits system $[10,11,17]$. In this paper, the entanglement values we calculate are global entanglement.

\section{ENTANGLEMENT In SHOR'S FACTORING ALgORITHM}

When the input quantum state of the second register in quantum phase estimation algorithm is a superposition state of eigenstates, the final state resulting from the first stage can be described as

$$
\begin{aligned}
& \frac{a_{1}}{2^{t / 2}} \sum_{k=0}^{2^{t}-1} e^{2 \pi i \varphi_{0} k}|k\rangle \otimes\left|u_{0}\right\rangle+\ldots+\frac{a_{m}}{2^{t / 2}} \sum_{k=0}^{2^{t}-1} e^{2 \pi i \varphi_{m} k}|k\rangle \otimes\left|u_{m}\right\rangle \\
= & \frac{1}{2^{t / 2}} \sum_{d=0}^{m} \sum_{k=0}^{2^{t}-1} a_{d} e^{2 \pi i \varphi_{d} k}|k\rangle \otimes\left|u_{d}\right\rangle
\end{aligned}
$$


here, $t$ is the qubit number of the first register, $m$ is the number of eigenvectors. Each eigenvector is written as $\left|u_{d}\right\rangle$ and the corresponding phase is $\varphi_{d}$. For shor's factoring algorithm, like introduces in Sec. 2.1, we can see

$$
\begin{aligned}
& \left|u_{d}\right\rangle=\frac{1}{\sqrt{r}} \sum_{k=0}^{r-1} e^{\frac{-2 \pi i d k}{r}}\left|x^{k} \bmod N\right\rangle, \\
& \varphi_{d}=d / r, \\
& a_{d}=1 / \sqrt{r}, \quad 0 \leqslant d \leqslant r-1 .
\end{aligned}
$$

Then according to Eq.(8), we obtain the final state resulting from the first stage of Shor's factoring algorithm

$$
\begin{aligned}
\left|\psi_{\text {stage } 1}\right\rangle & =\frac{1}{2^{t / 2}} \sum_{d=0}^{r-1} \sum_{k=0}^{2^{t}-1} \frac{1}{\sqrt{r}} e^{2 \pi i \frac{d}{r} k}|k\rangle \otimes \frac{1}{\sqrt{r}} \sum_{c=0}^{r-1} e^{-\frac{2 \pi i d c}{r}}\left|x^{c} \bmod N\right\rangle \\
& =\frac{1}{r 2^{t / 2}} \sum_{d=0}^{r-1} \sum_{k=0}^{2^{t}-1} e^{2 \pi i \frac{d}{r} k}|k\rangle \otimes \sum_{c=0}^{r-1} e^{-\frac{2 \pi i d c}{r}}\left|x^{c} \bmod N\right\rangle \\
& =\frac{1}{r 2^{t / 2}} \sum_{d=0}^{r-1} \sum_{k=0}^{2^{t}-1} \sum_{c=0}^{r-1} e^{2 \pi i d(k-c) / r}\left|k\left(x^{c} \bmod N\right)\right\rangle .
\end{aligned}
$$

Because

$$
\sum_{k=0}^{r-1} e^{\frac{2 \pi i n k}{r}}= \begin{cases}r, & n=m r, m=0,1,2 \ldots \\ 0, & \text { else }\end{cases}
$$

So according to Eq.(10, 11), we can obtain exact formula of amplitude of $\left|\psi_{\text {stage } 1}\right\rangle\left(b_{i}\right)$ is

$$
b_{i}= \begin{cases}\frac{1}{2^{t / 2}}, & k-c=r n, i=k^{*} 2^{q u N u m 2}+x^{c} \operatorname{modN} \\ 0, & \text { else }\end{cases}
$$

here $n=0,1,2 \ldots, 0, c, r-1$, "quNum2" is the qubit number in the second register. 


\subsection{Algorithm generate no entanglement}

From Eq.(12), we obtain in the case when $r=1,\left|\psi_{\text {stage } 1}\right\rangle$ is

$$
\left(0, \frac{1}{2^{t / 2}}, 0, \frac{1}{2^{t / 2}}, \ldots, 0, \frac{1}{2^{t / 2}}\right)^{\dagger}
$$

To obtain global entanglement, we need to calculate all bipartite entanglement of the n-qubits system. The bipartition can be classified by row bits as (1) all of them are in the first register, (2) all of them are in the second register, (3) part of them are in the first register, others are in the second register. Now we calculate bipartite entanglement in these three cases.

In the first case when all of the row bits of the coefficient matrix are in the first register, the coefficient matrix can be written as

$$
\left(\begin{array}{ccccccc}
0 & \frac{1}{2^{t / 2}} & 0 & \frac{1}{2^{t / 2}} & \ldots & 0 & \frac{1}{2^{t / 2}} \\
0 & \frac{1}{2^{t / 2}} & 0 & \frac{1}{2^{t / 2}} & \ldots & 0 & \frac{1}{2^{t / 2}} \\
\vdots & \vdots & \vdots & \vdots & \vdots & \vdots & \vdots \\
0 & \frac{1}{2^{t / 2}} & 0 & \frac{1}{2^{t / 2}} & \ldots & 0 & \frac{1}{2^{t / 2}}
\end{array}\right)
$$

Then in the second case when all of the row bits of the coefficient matrix are in the second register, the coefficient matrix can be written as

$$
\left(\begin{array}{ccccccc}
0 & 0 & 0 & 0 & \ldots & 0 & 0 \\
\frac{1}{2^{t / 2}} & \frac{1}{2^{t / 2}} & \frac{1}{2^{t / 2}} & \frac{1}{2^{t / 2}} & \cdots & \frac{1}{2^{t / 2}} & \frac{1}{2^{t / 2}}
\end{array}\right)
$$

Finally, in the case when part of the row bits of the coefficient matrix are in the second register, others are in the second register, the coefficient matrix can be written as

$$
\left(\begin{array}{ccccccc}
0 & 0 & 0 & 0 & \cdots & 0 & 0 \\
\frac{1}{2^{t / 2}} & \frac{1}{2^{t / 2}} & \frac{1}{2^{t / 2}} & \frac{1}{2^{t / 2}} & \cdots & \frac{1}{2^{t / 2}} & \frac{1}{2^{t / 2}} \\
0 & 0 & 0 & 0 & \cdots & 0 & 0 \\
\frac{1}{2^{t / 2}} & \frac{1}{2^{t / 2}} & \frac{1}{2^{t / 2}} & \frac{1}{2^{t / 2}} & \cdots & \frac{1}{2^{t / 2}} & \frac{1}{2^{t / 2}} \\
\vdots & \vdots & \vdots & \vdots & \vdots & \vdots & \vdots \\
0 & 0 & 0 & 0 & \cdots & 0 & 0 \\
\frac{1}{2^{t / 2}} & \frac{1}{2^{t / 2}} & \frac{1}{2^{t / 2}} & \frac{1}{2^{t / 2}} & \cdots & \frac{1}{2^{t / 2}} & \frac{1}{2^{t / 2}}
\end{array}\right)
$$

According to equality condition for Cauchy-Schwarz inequality, for these three cases, the bipartite entanglement are all 0. 
Global entanglement of an n-qubit state is the sum of the bipartite entanglement measures associated with all possible bipartitions of the n-qubits system. So when $r=1$, Shor's factoring algorithm generate no entanglement.

\subsection{Algorithm generate entanglement}

The global amount of entanglement exhibited by an $n$-qubit state is provided by the sum of the bipartite entanglement measures associated with all possible bipartitions of the n-qubits system. So if there is at least one bipartite entanglement is not 0 , the global entanglement definitely not 0 , the algorithm will generate entanglement. When we group by qubit in the first register, $\left|\psi_{\text {stagel }}\right\rangle$ can be rewrite as

$$
\begin{aligned}
\left|\psi_{\text {stagel }}\right\rangle & =\left(B^{(0)}, B^{(1)}, \ldots, B^{\left(2^{t}-1\right)}\right)^{?}, \\
B_{i}^{(k)} & = \begin{cases}\frac{1}{2^{t / 2}}, & i=x^{k} \bmod N . \\
0, & \text { else }\end{cases}
\end{aligned}
$$

$B^{(k)}$ is periodic with period $\mathrm{r}$, that

$$
B^{(l)}=B^{(l+n r)}, \quad \begin{gathered}
n=0,1,2 \ldots, \\
l=0,1, \ldots, \quad l<r
\end{gathered}
$$

Obvious, when $\mathrm{r}=1, B^{(0)}=B^{(1)}=\ldots=B^{\left(2^{t}-1\right)}=\left(0, \frac{1}{2^{t / 2}}\right)$. But in the case when $r \neq 1$, we will find $B^{\left(l_{i}+n r\right)} \neq B^{\left(l_{j}+n r\right)}, i \neq j$. Then we will certainly find a coefficient matrix, elements of which satisfy $c_{d k}=B^{\left(l_{i}+n r\right)}, c_{e k}=B^{\left(l_{j}+n r\right)}, i \neq j, d \neq e$. The bipartite entanglement based on this coefficient matrix is not 0 . So Shor's factoring algorithm will generate entanglement when $r \neq 1$. To understand it more clearly we give an example in Appendix. A.

\section{DISCUSSION}

Theoretically, in two cases: when the input state of the second register is an explicit eigenstate or the order $r=1$, Shor's algorithm will not generate entanglement. But in the real world, for the first case, to find an explicit eigenstate to use as input is as hard as finding the eigenvalue that the quantum algorithm aims to estimate. And the second case is meaningless. So the algorithm will always generate entanglement in practical works. Researchers have showed that most instances involve multipartite entanglement for Deutsch-Jozsa, Grover, and Simon algorithms [3, 8]. Our results give the similar results for Shor's factoring algorithm.

Different approaches to the analysis of the entanglement generated by Shor's factoring algorithm were presented in Refs. [5, 9]. In agreement with Ref. [5, 7], the entanglement is primarily 
generated during the first stage. Ref. [9] demonstrates that there is entanglement of an unbounded number of particles in Shor's algorithm and it gives a simple case in which the state is blocked.

$$
\begin{aligned}
\left|A P_{1}\right\rangle & =\sum_{k=0}^{3}|3+3 k\rangle=(|3\rangle+|6\rangle+|9\rangle+|12\rangle) \\
& \left.\left.\left.\left.=0_{3} 0_{2} 1_{1} 1_{0}\right\rangle+0_{3} 1_{2} 1_{1} 0_{0}\right\rangle+1_{3} 0_{2} 0_{1} 1_{0}\right\rangle+1_{3} 1_{2} 0_{1} 0_{0}\right\rangle . \\
& =\left(\left|0_{3} 1_{1}\right\rangle+\left|1_{3} 0_{1}\right\rangle\right)\left(\left|0_{2} 1_{0}\right\rangle+\left|1_{2} 0_{0}\right\rangle\right)
\end{aligned}
$$

For this point, Ref. [9] neglect "partially separable" (a "structural" property of the entanglement of the states [3]). It is easy to find although the state can be written as the form of product state, but there exist partially entanglement, that is the entanglement between qubit 0 and qubit 2 and also the entanglement between qubit 1 and qubit 3. Like Ref. [10] the entanglement measure we use is a "global measure" [10], which naturally involve all qubits in the register of the algorithm. And we prove Shor's algorithm will always generate entanglement except two cases: when the input state of the second register is an explicit eigenstate or $r=1$.

Ref. [5] gives conclusion that for some values of $\mathrm{N}$ and choices of $\mathrm{x},(1) G C D(N, x) \neq 1$, (2)

$x^{2^{n}}=1 \bmod N$, Shor's algorithm produces no entanglement. There is a simple counter-example that $\mathrm{x}=2, \mathrm{~N}=3, \mathrm{r}=2$. The corresponding periodic state $[5,7]$ of $\mathrm{q}=3$ qubits, with period $\mathrm{r}=2$ and shift $1=3$ is

$$
\begin{aligned}
\left|\psi_{2,3}^{3}\right\rangle & =\frac{1}{\sqrt{3}} \sum_{j=0}^{2}|3+2 j\rangle \\
& =\frac{1}{\sqrt{3}}(|3\rangle+|5\rangle+|7\rangle) \\
& =\frac{1}{\sqrt{3}}(|011\rangle+|101\rangle+|111\rangle)
\end{aligned} .
$$

Obviously, $\left|\psi_{2,3}^{3}\right\rangle$ is a entangled state. But the state meets the condition (2). It should be a product state on the conclusion in Ref. [5]. We suspect that this is because the entanglement measure Ref. [5] used is Groverian entanglement measure. From the mathematical viewpoint Groverian measure turns the entanglement solving problem into the constrained optimization problem. Ref. [5] gives an algorithm for obtaining the entanglement, but it easily runs into the local optimization solution. Ref. [18] gives an explanation for this. Besides, the quantum states Refs. [5, 9] focused were states in the first register after the measurement, but not all the states (both including in the first register and the second register) the algorithm involved. In the first stage, the algorithm acts on all the states and before measurement, all the states may entangled together. We prove in practical works the algorithm will always generate entanglement before measurement.

\section{A. Appendix: Example of $r=1$}

In the case $\mathrm{x}=2, \mathrm{~N}=3$, then $\mathrm{r}=2$. When we set qubit number in the first register is 2 , at this time we can get 


$$
\begin{aligned}
& \left|\psi_{\text {stage } 1}\right\rangle=
\end{aligned}
$$

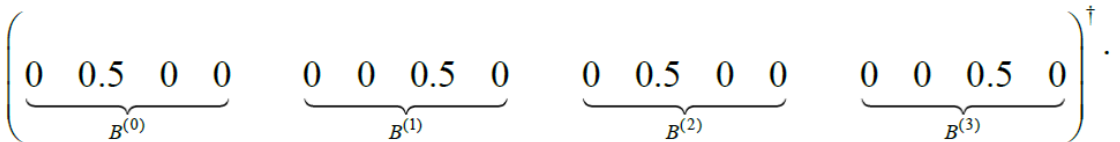

Obvious, $B^{(0)}=B^{(2)}, B^{(1)}=B^{(3)}$. When we choose the second qubit is row bit, the corresponding coefficient matrix is

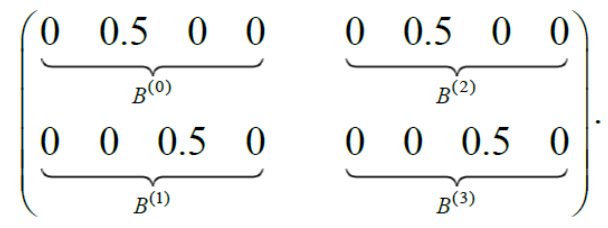

In this coefficient matrix $c_{00}=B^{(0)}, c_{10}=B^{(1)}, c_{00} \neq c_{10}$. The corresponding bipartite entanglement is not 0 . So in this case, the global entanglement is not 0 . Shor's factoring algorithm will generate entanglement.

\section{ACKNOWLEDGEMENTS}

This work is supported by the National Natural Science Foundation of China (Grant No. 61170321,61502101,61871120,61802002), Natural Science Foundation of Jiangsu Province, China (Grant No. BK20140651), Natural Science Foundation of Anhui Province, China (Grant No. 1608085MF129), Research Fund for the Doctoral Program of Higher Education (Grant No. 20110092110024), Foundation for Natural Science Major Program of Education Bureau of Anhui Province (Grant No. KJ2015ZD09) and the open fund of Key Laboratory of Computer Network and Information Integration in Southeast University, Ministry of Education, China (Grant No. K93-9-2015-10C).

\section{REFERENCES}

[1] Shantanav Chakraborty, Subhashish Banerjee, Satyabrata Adhikari, and Atul Kumar. Entanglement in the grover's search algorithm. arXiv preprint arXiv:1305.4454, 2013.

[2] Arti Chamoli and Bhandari C. Groverian entanglement measure and evolution of entanglement in search algorithm for $\mathrm{n}(=3,5)$-qubit systems with real coefficients. Quantum Information Processing, 6(4):255- 271, 2007.

[3] Ri Qu, Bingjian Shang, Yanru Bao, Dawei Song, ChunMing Teng, and Zhiwei Zhou. Multipartite entanglement in grovers search algorithm. Natural Computing, 14(4):683-689, 2015.

[4] M. Rossi, D. Bru Ss, and C. Macchiavello. Scale invariance of entanglement dynamics in grover's quantum search algorithm. Physical Review A, 87(2):022331, 2013.

[5] Yishai Shimoni, Daniel Shapira, and Ofer Biham. Entangled quantum states generated by shors factoring algorithm. Physical Review A, 72(6):062308, 2005.

[6] Vivien M. Kendon and William J. Munro. Entanglement and its role in shor's algorithm. arXiv preprint quant-ph/0412140, 2004.

[7] Yonatan Most, Yishai Shimoni, and Ofer Biham. Entanglement of periodic states, the quantum fourier transform, and shor's factoring algorithm. Phys. Rev. A, 81:052306, 2010. 
[8] D. Bru Ss and C. Macchiavello. Multipartite entanglement in quantum algorithms. Physical Review A, 83(5):052313, 2011.

[9] Richard Jozsa and Noah Linden. On the role of entanglement in quantum-computational speed-up, 2003.

[10] J. Batle, CH Raymond Ooi, Ahmed Farouk, M. S. Alkhambashi, and S. Abdalla. Global versus local quantum correlations in the grover search algorithm. Quantum Information Processing, 15(2):833849, 2016.

[11] Chao Zhao and Yang Guo-wu. A multipartite entanglement measure based on coefficient matrices. Quantum Information Processing, 14(8):2861-2881, 2015.

[12] Michael A. Nielsen and Isaac L. Chuang. Quantum computation and quantum information. Cambridge university press, 2010.

[13] Guifr E. Vidal. Efficient classical simulation of slightly entangled quantum computations. Physical Review Letters, 91(14):147902, 2003.

[14] Xiang Zhan, Xin Zhang, Jian Li, Yongsheng Zhang, Barry C. Sanders, and Peng Xue. Realization of the contextuality-nonlocality tradeoff with a qubit-qutrit photon pair. Phys. Rev. Lett., 116:090401, 2016.

[15] Xiangrong Li and Dafa Li. Classification of general n-qubit states under stochastic local operations and classical communication in terms of the rank of coefficient matrix. Phys. Rev. Lett., 108:180502, 2012.

[16] Xiangrong Li and Dafa Li. Method for classifying multiqubit states via the rank of the coefficient matrix and its application to four-qubit states. Phys. Rev. A, 86:042332, 2012.

[17] Vineeth S. Bhaskara and Panigrahi. Generalized concurrence measure for faithful quantification of multiparticle pure state entanglement using lagrange's identity and wedge product. Quantum Information Processing, 16(5):118, 2017.

[18] Alexander Streltsov, Hermann Kampermann, and Dagmar Bru Ss. Simple algorithm for computing the geometric measure of entanglement. Phys. Rev. A, 84:022323, 2011. 